\title{
A novel multikinase inhibitor R8 exhibits potent inhibition on cancer cells through both apoptosis and autophagic cell death
}

\author{
Yuqiong Xie ${ }^{1, *}$, Chunchun $\mathrm{Li}^{1,{ }^{1}}$, Yali Huang ${ }^{2}$, Zhenyu $\mathrm{Jia}^{3}$ and Jiang $\mathrm{CaO}^{1}$ \\ ${ }^{1}$ Clinical Research Center, The Second Affiliated Hospital, Zhejiang University School of Medicine, Hangzhou 310009, China \\ ${ }^{2}$ Institute of Hygiene, Zhejiang Academy of Medical Sciences, Hangzhou 310013, China \\ ${ }^{3}$ Institute of Occupational Diseases, Zhejiang Academy of Medical Sciences, Hangzhou 310013, China \\ *These authors have contributed equally to this work \\ Correspondence to: Jiang Cao, email: caoj@zju.edu.cn \\ Zhenyu Jia, email: zhenyujia@yahoo.com
}

Keywords: multikinase inhibitor, targeted therapy, cancer, autophagy, apoptosis

Received: May 19, $2017 \quad$ Accepted: July 13, $2017 \quad$ Published: August 14, 2017

Copyright: Xie et al. This is an open-access article distributed under the terms of the Creative Commons Attribution License 3.0 (CC BY 3.0), which permits unrestricted use, distribution, and reproduction in any medium, provided the original author and source are credited.

\section{ABSTRACT}

Chemotherapy is an important treatment for cancer patients, especially for those with unresectable lesions. Targeted therapy of cancer by specific inhibition of aberrant tyrosine kinase activities in cancer cells with chemically synthesized tyrosine kinase inhibitors (TKIs), shows better responses while less side effects than traditional chemotherapeutic drugs. It is common that cancer cells often exhibit deregulation of several tyrosine kinases simultaneously, multikinase TKIs (MKIs) therefore have greater advantages over single-target TKIs. Currently more MKIs are under developing for better efficacy for different types of cancer. In the present work, we evaluated the in vitro therapeutic potential of a novel MKI, namely R8, with comparison to the clinically available MKI Sunitinib. Results showed that $\mathbf{R} 8$ has stronger inhibition on six different types of cancer cell lines with lower IC $_{50}$ than Sunitinib does. Cell cycle analysis showed that R8 induced significant G0/G1 arrest phase of lung cancer A549 and NCI-H226 cells. The inhibition was also confirmed by colony formation and migration assays in both lung cancer cell lines in a dose-dependent manner. R8 could significantly inhibit the phosphorylation of multiple receptor tyrosine kinases (RTKs) included PDGFR $\beta$, VEGFR2, EGFR and C-Kit, leading to the down-regulation of PI3K-Akt-mTOR signaling. Further analysis revealed that $\mathbf{R} 8$ treatment induced more significant apoptosis than Sunitinib did, which might be the consequence of the autophagic cell death. In conclusion, this work suggested $R 8$ to be a promising novel anticancer MKI, and provided the basis for further in vivo investigation on its potential in treatment of lung cancer.

\section{INTRODUCTION}

Cancer is one of the leading causes of death worldwide, with an estimated 14.1 million new cases and 8.2 million deaths occurred in 2012 worldwide [1]. Chemotherapy is an important way of treatment for cancer patients, especially for those with unresectable lesions. Targeted therapy of cancer by specific inhibition of aberrantly activated receptor tyrosine kinase (RTK) activities in cancer cells with chemically synthesized small molecule compounds, tyrosine kinase inhibitors (TKIs), shows better responses while less side effects than traditional chemotherapeutic drugs. It is common that cancer cells often exhibit simultaneous deregulation of several tyrosine kinases, such as epithelial growth factor receptor (EGFR), platelet-derived growth factor receptor (PDGFR), vascular endothelial growth factor receptor (VEGFR), etc., which lead to the malignant characteristics 
of tumor such as uncontrolled proliferation, invasiveness and angiogenesis, multi-target TKIs (or multikinase inhibitors, MKIs) therefore have great advantages over single-target TKIs [2].

Although many TKIs/MKIs have been widely used clinically in the treatment of various types of cancer, they do not always give satisfactory outcomes, even for individuals with definite hyper-activated target kinases. Primary resistance and acquired resistance are commonly observed because of the fact that, each type of cancer or even each individual cancer cell may show different aberrantly activated tyrosine kinases due to heterogenicity during the development and progression of the disease, and the TKIs/MKIs may also have different binding and inhibition selectivities on different kinases (and even same kinase with different mutations) [3-5]. Therefore more multi-target TKIs are developed or under developing for better efficacies on different individuals and to overcome the primary or acquired resistance [6-8].

In the present work, we evaluated the in vitro inhibitory effect of a novel multikinase inhibitor, namely R8 (Figure 1), on several different types cancer cells. We further focused on the mechanism for its inhibition on lung cancer cells specifically, which showed distinct characteristics compared to Sunitinib, a clinically available MKI which has been investigated extensively in many types of cancer including lung cancer, either used alone or in combination with other therapeutics [9-11].

\section{RESULTS}

\section{R8 significantly inhibited proliferation of non- small lung cancer cell line in a dose-dependent manner}

In order to evaluate efficacy of R8 on inhibiting cell proliferation, we selected clinically available MKI Sunitinib as a control, which was reported to be successful in inhibiting proliferation of variety kinds of tumors. We selected six cell lines, list as follows: human gastric carcinoma cells SGC7901, non-small lung cancer cell line A549 and NCI-H226, laryngeal carcinoma cell line HEp2 , renal adenocarcinoma cell line 786-0, colorectal cancer cell line SW620. As indicated by the cell viability curves (Figure 2A-Figure 2F), both Sunitinib and R8 inhibited proliferation of six cell lines in a dose-dependent manner. Surprisingly, R8 showed lower $\mathrm{IC}_{50}$ values than Sunitinib. The $\mathrm{IC}_{50}$ values of $\mathrm{R} 8$ were 9.61, 3.80, 6.78, 4.88, 2.25 and $4.05 \mu \mathrm{M}$, whereas $\mathrm{IC}_{50}$ values of Sunitinib were 15.01, 7.04, 28.11, 7.58, 13.75 and $10.14 \mu \mathrm{M}$ in SGC7901, A549, NCI-H226, HEp-2, 786-0 and SW620 cell lines, respectively.

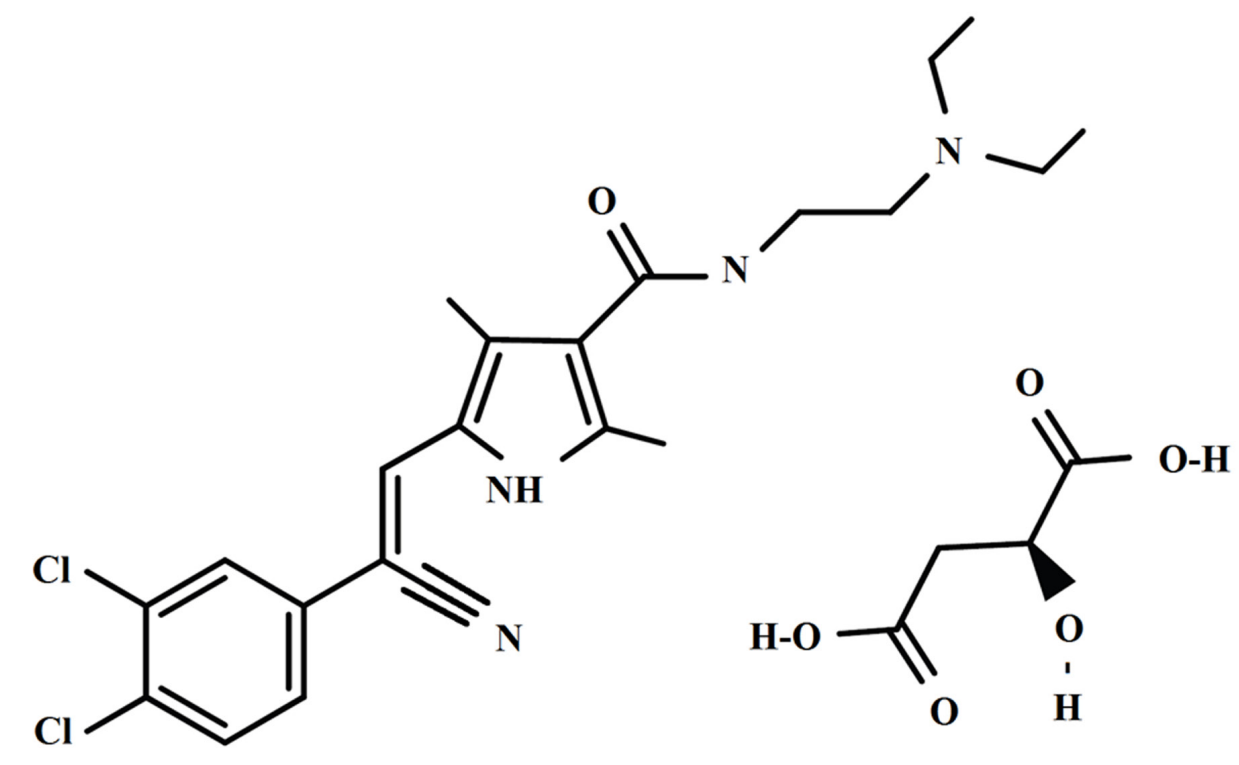

R8

Chemical Formula: $\mathrm{C}_{22} \mathrm{H}_{26} \mathrm{Cl}_{2} \mathrm{~N}_{4} \mathrm{O} \cdot \mathrm{C}_{4} \mathrm{H}_{6} \mathrm{O}_{5}$

Molecular Weight: 567.46

Figure 1: Chemical structure of R8. 
A
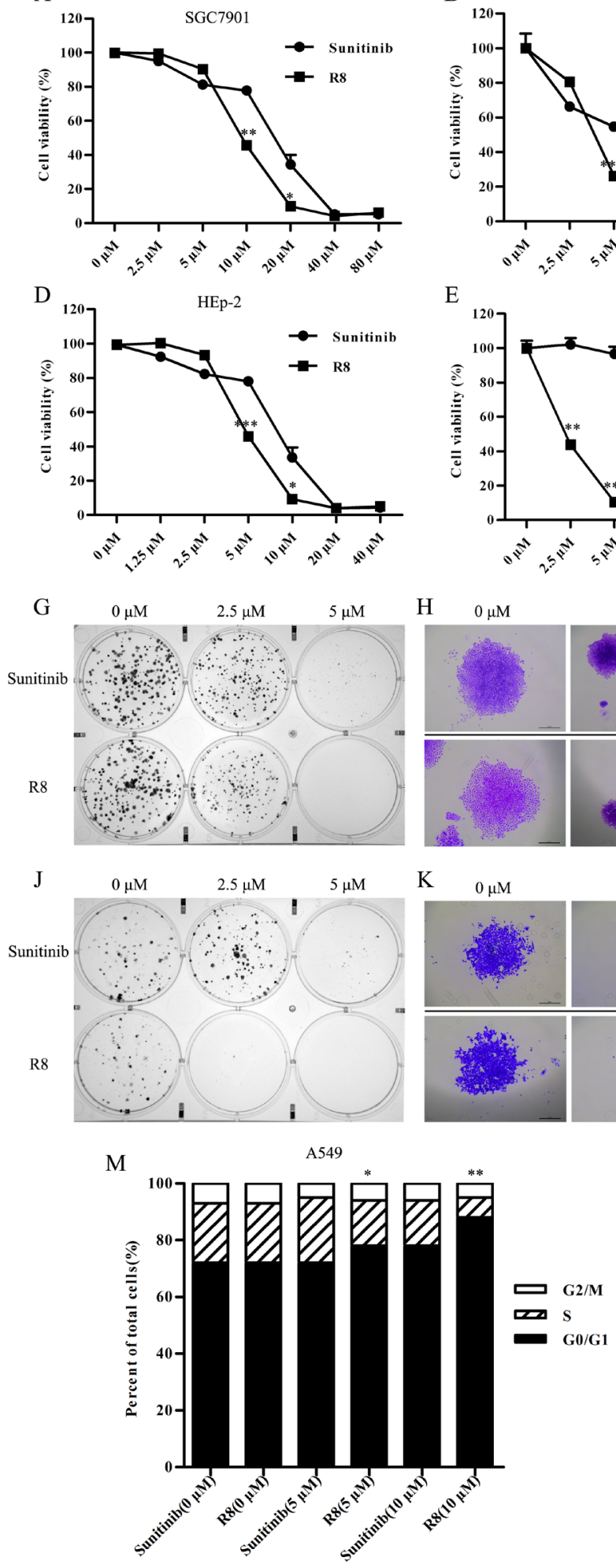

B

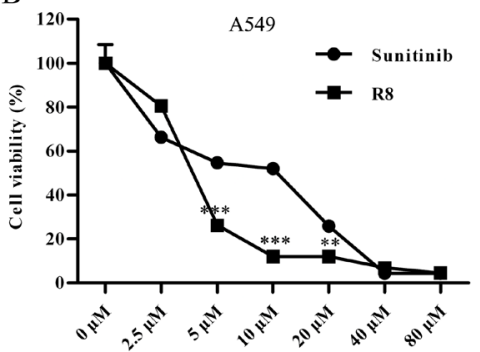

E

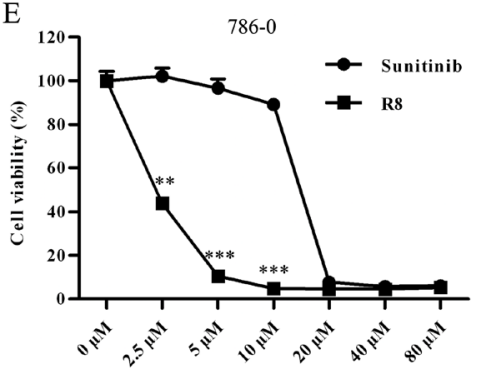

$\mathrm{H} \quad 0 \mu \mathrm{M} \quad 2.5 \mu \mathrm{M} \quad 5 \mu \mathrm{M}$

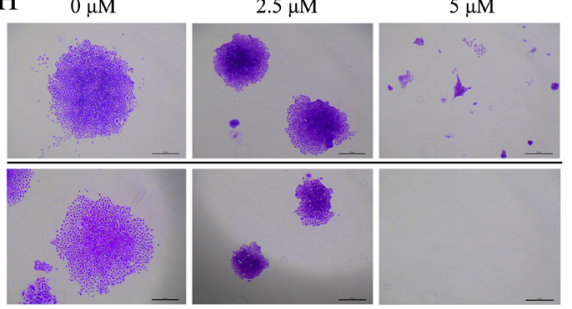

K

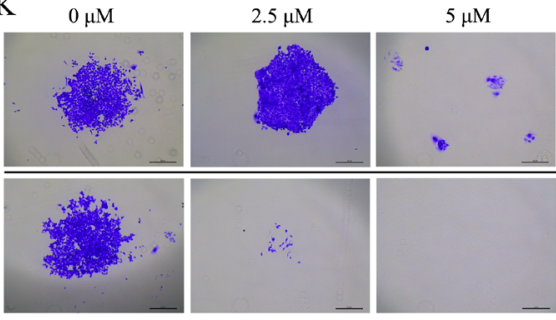

$\mathrm{C}$

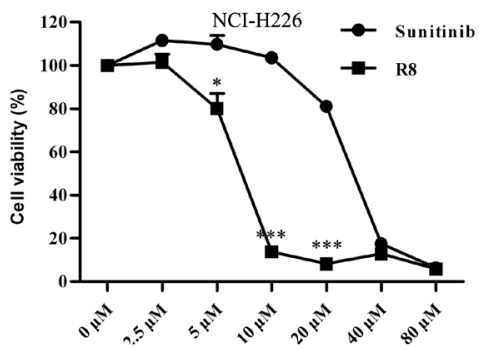

F

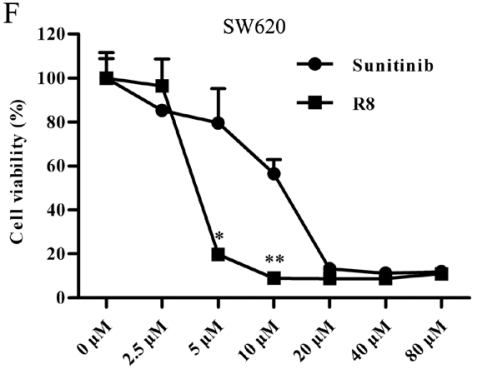

I

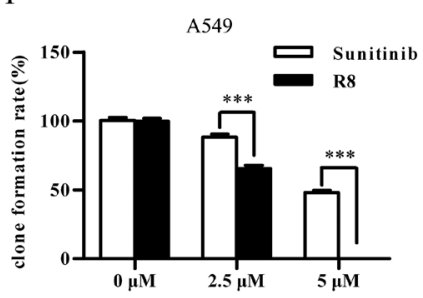

$\mathrm{L}$

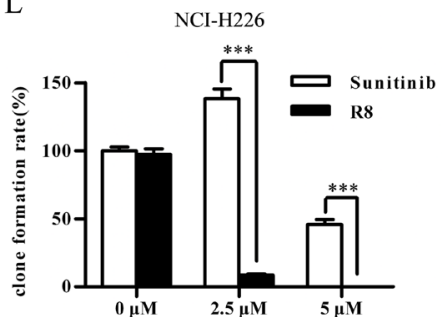

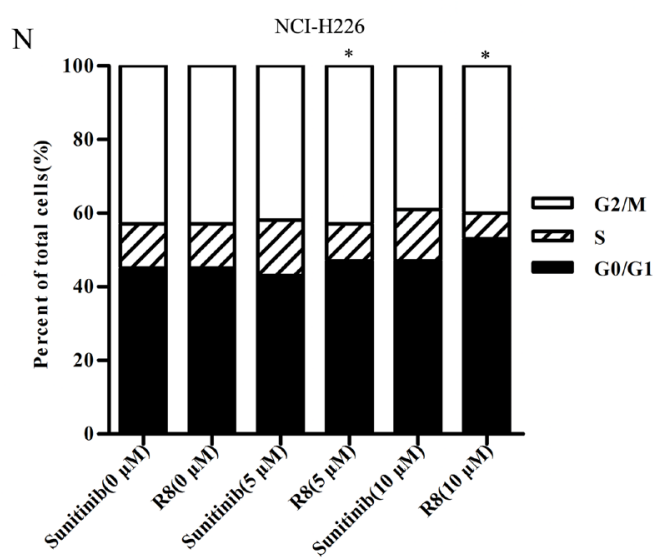

Figure 2: R8 significantly inhibited cell proliferation and colony formation than Sunitinib. (A, F) Cell viability was detected by CCK-8 assay after six cell lines treated with Sunitinib or R8 for 48 h. (A) SGC7901, (B) A549, (C) NCI-H226, (D) HEp-2, (E) 786-0, (F) SW620. (G, J) R8 treatment of A549 (G) and NCI-H226 (J) significantly decreased numbers of colonies than Sunitinib after 14 days culture at concentration of $2.5 \mu \mathrm{M}$ and $5 \mu \mathrm{M}$. (H, K) Typical colony morphology of A549 (H) and NCI-H226 (K) generated from single cell. Scale bar $=20 \mu \mathrm{m}$. (I, L) Colony formation rate analysis of A549 (I) and NCI-H226 (L) treated with Sunitinib or R8. (M, N) Higher G0/G1 phase cell cycle arrest was induced after A549 and NCI-H226 cell lines treated with R8 for $24 \mathrm{~h}$ than Sunitinb. Data were presented as means $\pm \mathrm{SD}$ of three independent experiments $\left({ }^{*} P<0.05,{ }^{* *} P<0.01,{ }^{* * *} P<0.001\right)$. 
Furthermore, the long-term effect of R8 on cell survival was determined by colony formation assay. The results further confirmed that $\mathrm{R} 8$ was more significantly in inhibiting the proliferation of both A549 (Figure 2GFigure 2I) and NCI-H226 (Figure 2J-Figure 2L) than Sunitinb.

\section{R8 induced G0/G1-phase cell cycle arrest and inhibited cell migration in non-small lung cancer cells}

To identify the mechanism underlying R8-mediated inhibition of proliferation, cell cycle analysis was performed. R8 caused enhanced accumulation of cells in G1 phase and decrease in S phase than Sunitinib in A549 (Figure 2M) and NCI-H226 (Figure 2N). Significantly induction of $\mathrm{G} 0 / \mathrm{G} 1$ phase arrest may be one of reasons to answer why R8 was more competent in inhibiting cell proliferation. Since migration and angiogenesis are essential for tumor metastasis, so next we investigated whether R8 could inhibit cell migration ability. As shown in Figure 3, R8 significantly inhibited NCI-H226 (Figure $3 \mathrm{~A}$, Figure 3B) and A549 (Figure 3C) migration in a dose- dependent manner than Sunitinib. But when we evaluated tube formation of HUVECs treated with Sunitinib or R8, R8 was less efficient in inhibiting VEGF pathway than Sunitinib (Figure 3D, Figure 3E).

\section{R8 inhibited growth factor stimulated receptor phosphorylation}

Above data indicated R8 could inhibit A549 and NCI-H226 cells proliferation and migration, but the $\mathrm{IC}_{50}$ values in two cell lines were different, NCI-H226 cell line seemed more resistant to drug treatment. So next we compared the expression levels of PDGFR $\beta$, VEGFR2, EGFR, C-kit between A549 and NCI-H226. Undoubtedly, NCI-H226 expressed high level of totally four receptors, especially for EGFR and PDGFR $\beta$, whereas A549 only with medium level of EGFR and low level of C-Kit expression (Figure 4A).

So next, we evaluated the phosphorylated status of tyrosine kinase receptors in A549 and NCI-H226 after exposed to Sunitinib or R8 for $1 \mathrm{~h}$, respectively. Sunitinib treatment showed slight decrease in p-EGFR but no change with p-C-Kit, whereas R8 (10 $\mu \mathrm{M})$ treated
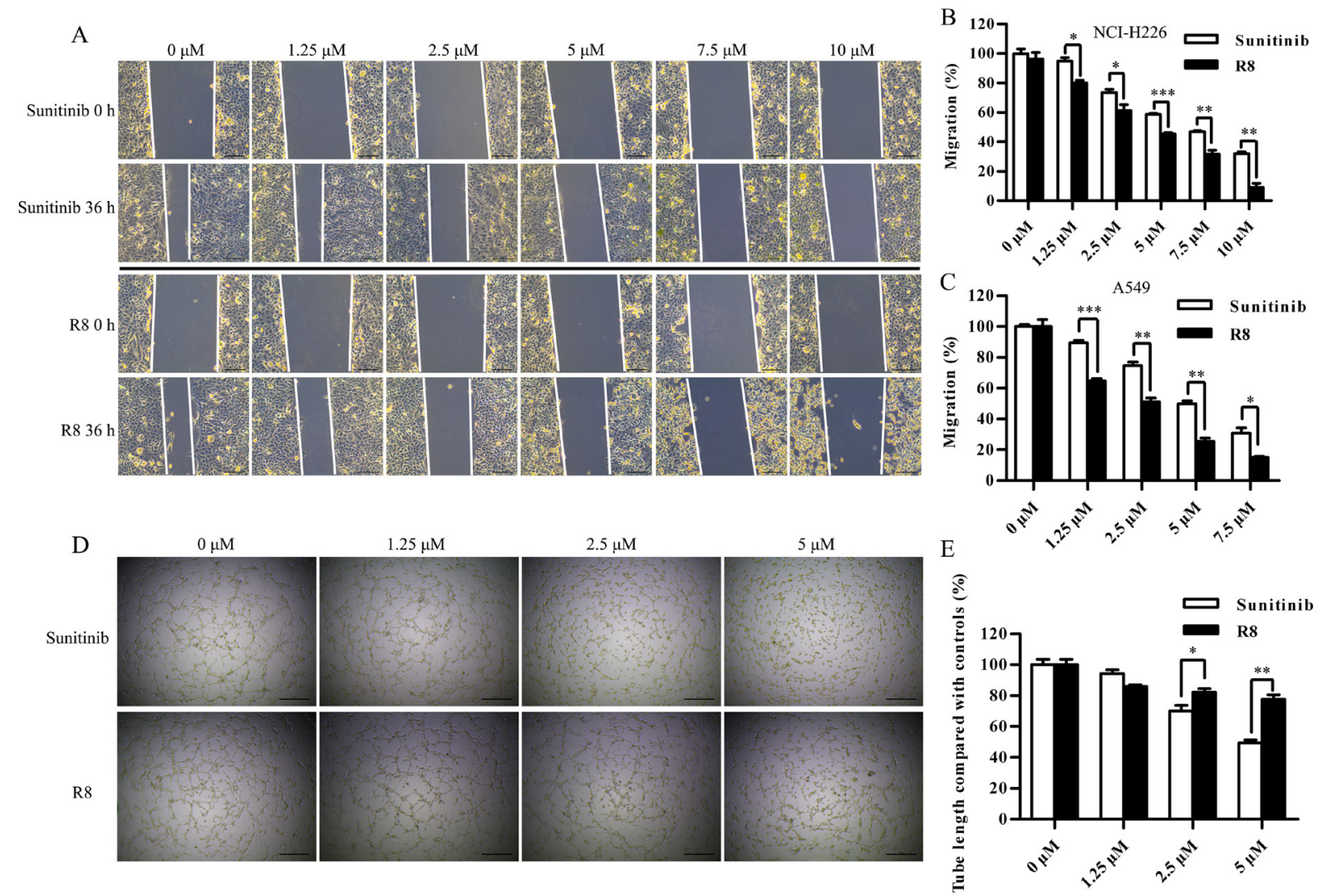

Figure 3: R8 inhibited cell migration of A549 and NCI-H226, but was less efficient in inhibiting tube formation. (A) Micrographs showed the partial closing gap of NCI-H226 after treated with various concentrations of R8 or Sunitinib. Scale bar=10 $\mu$ m. (B, C) Statistic analysis of cell migration after NCI-H226 and A549 treated with different doses of R8 or Sunitinib for 36 h. (D, E) Tube formation assay of HUVECs treated with different doses of Sunitinib or R8. Data were presented as means \pm SD of three independent experiments $\left({ }^{*} P<0.05,{ }^{* *} P<0.01,{ }^{* * *} P<0.001\right)$. 
A549 caused an up-regulation in p-EGFR. P-AKT and p-ERK were activated after stimulation of $10 \%$ FBS, and Sunitinib inhibited phosphorylation of p-ERK in a dose-dependent manner whereas no change with p-AKT. Although R8 caused up-regulation of p-EGFR, dosedependent inhibition of p-AKT, total AKT, p-ERK, total ERK were observed in R8 treated A549 cell line (Figure 4B).

For NCI-H226, which expressed abundant receptors, $10 \%$ FBS stimulation for 10 min caused significant upregulation of four receptors. Sunitinib and R8 inhibited p-PDGFR $\beta$, p-VEGFR2, p-EGFR, $p$-C-Kit expression in a dose-dependent manner. Although Sunitinib and R8 downregulated p-EGFR and p-C-Kit, but was not so effective than $\mathrm{p}$-PDGFR $\beta$, p-VEGFR2.

P-Akt and total AKT were decreased in R8 (20 $\mu \mathrm{M})$ treated NCI-H226 cells, but only a slight decrease of p-AKT in Sunitinib $(20 \mu \mathrm{M})$ treated groups (Figure $4 \mathrm{C}$ ).

\section{R8 induced autophagy and apoptosis}

Since treated with Sunitinib and R8 caused a decline of phosphorylated receptors which may induce cell starvation, accompanied with down-regulated p-AKT, both of which may result in autophagy.

So next we analyzed autophagy and apoptosis related proteins by Western blotting. LC3-II was a good indicator of autophagosome formation. For A549, R8 (5 $\mu \mathrm{M}, 10 \mu \mathrm{M})$ induced significant increase of LC3-II than Sunitinib in a dose-dependent manner. Apoptosis related proteins, such as cleaved-caspase-3 and cleaved PARP, were observed after treated with Sunitinib or R8 for 48 $\mathrm{h}$, but no significant up-regulation of Bax, a pro-apoptotic molecule (Figure 5A). For NCI-H226, the expression of LC3-II was up-regulated in a dose-dependent manner both in sunitinb and R8 treated group, whereas R8 showed more potent in accumulating of LC3-II. Significant cleavage of
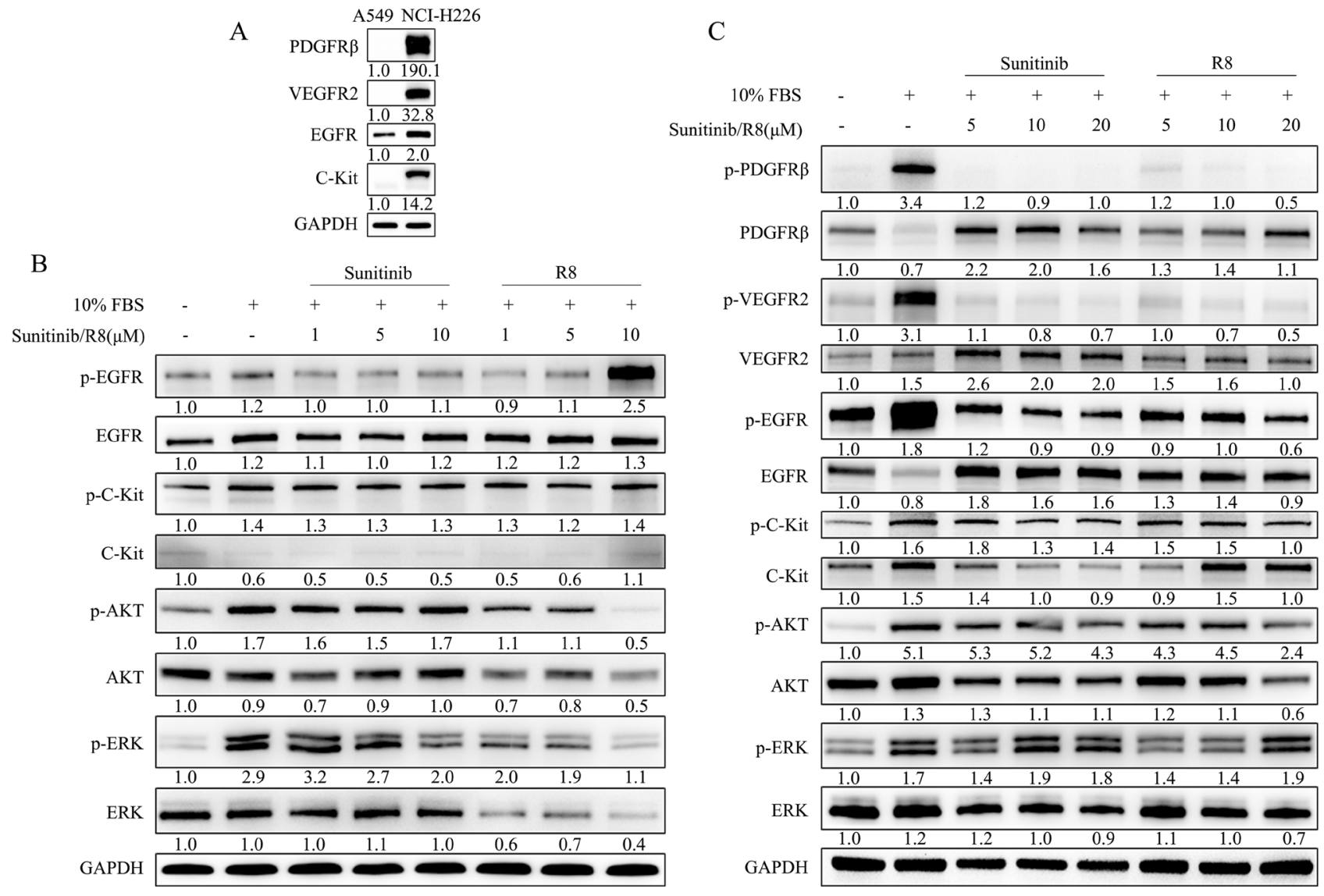

Figure 4: R8 significantly inhibited phosphorylation of receptor tyrosine kinases and down-stream AKT than Sunitinib in a dose-dependent manner. (A) Comparison of expression level of PDGFR $\beta$, VEGFR2, EGFR, C-Kit between A549 and NCI-H226 cell lines. All samples were $30 \mu \mathrm{g} / \mathrm{well}$, except for EGFR (20 $\mu \mathrm{g} / \mathrm{well})$. (B) A549 cell line was incubated with various concentration of R8 or Sunitinib for $1 \mathrm{~h}$, western blot analyzed phosphorylated EGFR, C-Kit, AKT, ERK after stimulation. (C) NCI-H226 cell line was incubated with various concentration of R8 and Sunitinib for $1 \mathrm{~h}$, western blot showed phosphorylated PDGFR $\beta$, VEGFR2, EGFR, C-Kit, AKT, ERK after stimulation. The protein densitometry values showed in the figure were normalized to their respective house-keeping protein GAPDH. 
caspase-3 and PARP, up-regulation of Bax were observed in $\mathrm{R} 8$ treated cells than Sunitinib (Figure 5B).

Since there were many vacuole-like bubbles in cytoplasm after NCI-H226 treated with R8 for $48 \mathrm{~h}$ compared with controls, so we performed LC3 immunofluorescence to distinguish cytosolic LC3-I and autophagosome-associated LC3-II. For NCI-H226 exposed to $20 \mu \mathrm{M}$ R8, red-punctate LC3-II was observed in the cytoplasm, which indicated the formation of autophagosome (Figure 5C).

\section{R8 induced type II programmed cell death}

In both A549 and NCI-H226 cell lines, R8 induced significant autophagy and apoptosis than Sunitinib. But the role of autophagy induced by Sunitinib or R8 was not
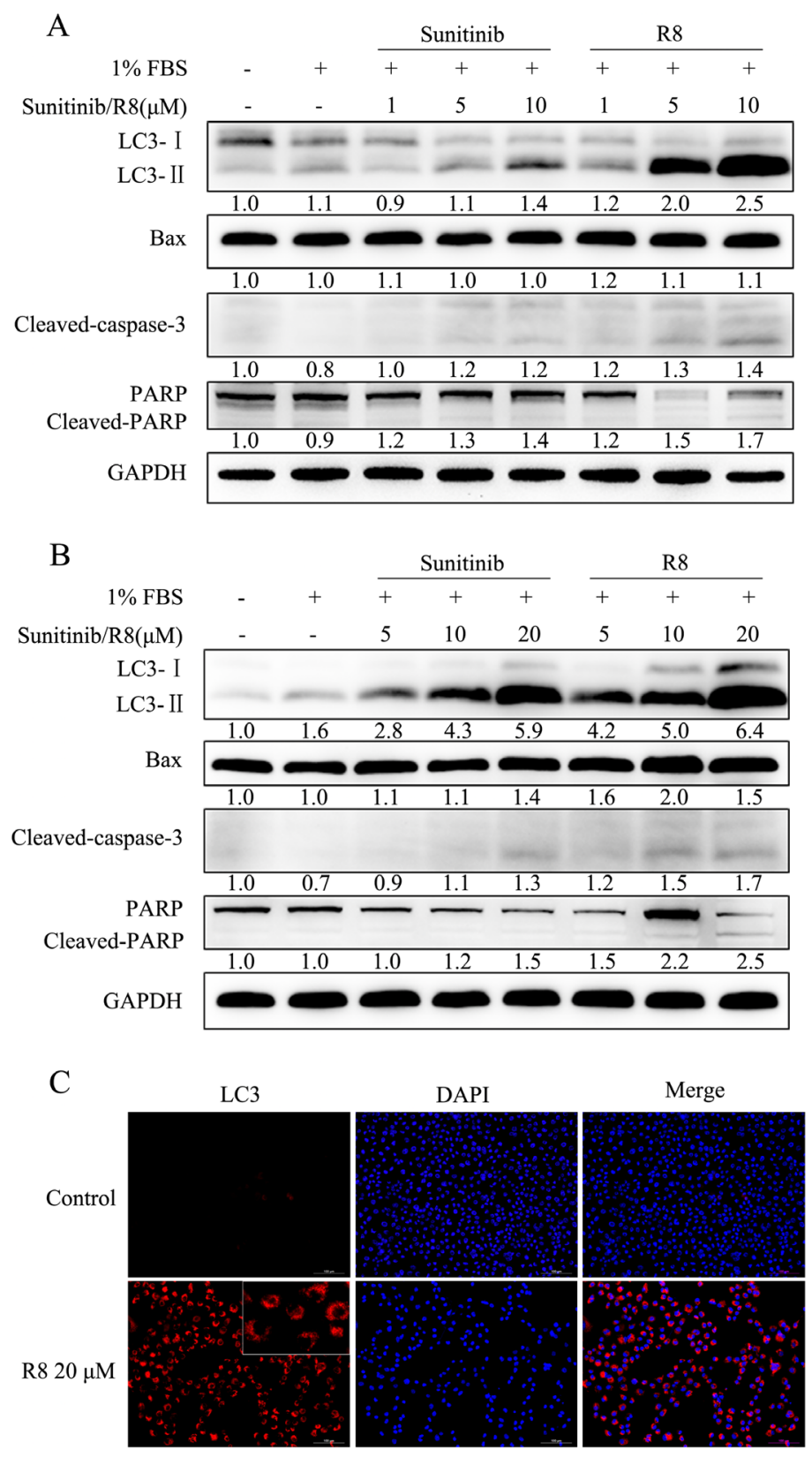

Figure 5: Strong autophagy was induced after A549 and NCI-H226 treated with high concentration of R8 for 48 h, as well as apoptosis. (A) A549 cell line treated with different doses of Sunitinib or R8 for $48 \mathrm{~h}$, western blotting analysis of the level of Bax, LC3, cleaved- caspase-3, PARP. GAPDH was used as a loading control. Experiments were repeated for three times and the data showed the representative results. (B) Significant activation of apoptosis and autophagy of NCI-H226 cell line was observed via western blotting of Bax, cleaved- caspase-3, PARP and LC3-II after treated with different doses of R8 for $48 \mathrm{~h}$, especially for the high concentration group. (C) Immunofluorescence of LC3 for NCI-H226 cell line treated with R8 for $48 \mathrm{~h}$. Red-punctate fluorescence of LC3-II could be detected in the $\mathrm{R} 820 \mu \mathrm{M}$ treated group. 
clear. So we first incubated cells with autophagy inhibitor 3-Methyladenine (3-MA) or chloroquine phosphate (CQ) before drug treatment.

For A549, inhibition of autophagy by 3-MA or CQ resulted in increasing of cleaved caspase-3 and PARP, which indicated autophagy induced by Sunitinib in A549 was a self-protected autophagy. Once inhibited autophagy, apoptosis would increase. On the other hand, for R8 treated cells, 3-MA pre-treatment resulted in decreasing of Beclin-1, LC3-II, cleaved caspase-3 and PARP, which indicated that the autophagy induced by $\mathrm{R} 8$ was deathleading (Figure 6A). Once inhibited autophagy, cell would survive. CQ pre-treated A549 only showed slight function in inhibiting cell-death autophagy, which indicated class III-PI3K was more important in R8-induced autophagy.

For NCI-H226, 3-MA or CQ treatment also enhanced Sunitinib induced apoptosis, marked by upregulation of cleaved caspase- 3 and PARP. In R8 treated cells, 3-MA treatment only decreased 19 KD cleaved caspase-3 and PARP without down-regulation of LC3-II. Whereas CQ treatment caused significant down-regulation of Beclin-1, cleaved caspase-3 and PARP (Figure 6B).
So in NCI-H226, CQ was more potent in inhibiting R8 induced autophagy, which indicated fusion with lysosome was more important in R8-induced autophagy of NCI-H226 cell line.

Besides, cell viability was up-regulated after A549 (Figure 6C) and NCI-H226 (Figure 6D) treated with combination of R8 and 3-MA, but not for the Sunitinib treated cells.

In conclusion, Sunitinib induced a self-protected autophagy, protected cells from apoptosis; whereas R8 induced autophagic cell death, which contributed to apoptosis.

\section{DISCUSSION}

In this study, we observed the inhibition of a novel MKI R8 on cancer cells in vitro, with comparison to Sunitinib, one of the widely used MKIs in clinic, and explored the possible mechanism by which R8 took effect. As a class III/V multikinase inhibitor, Sunitinib was reported to suppress various kinds of tumors, such as human metastatic renal cell carcinoma, lung carcinoma,
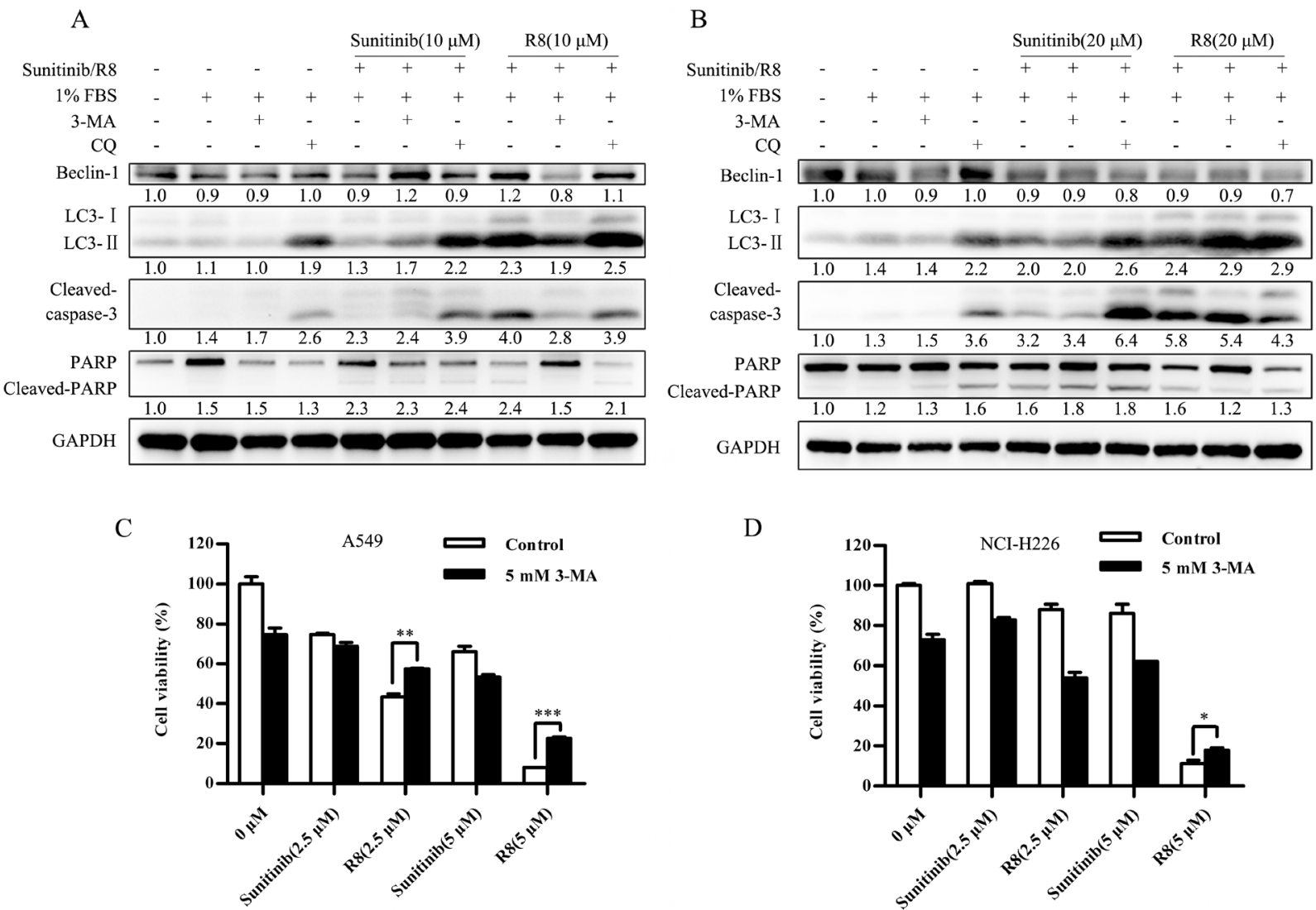

Figure 6: Self-protected role of Sunitinib induced autophagy whereas cell death mechanism of R8 induced autophagy. (A, B) Autophagy inhibitor 3-methyladenine (3-MA) and chloroquine (CQ) were used to evaluate the role of autophagy induced by Sunitinib and R8, respectively. Western blotting analysis of the level of Beclin-1, LC3, cleaved-caspase-3, PARP in A549 (A) and NCI-H226 (B) cell line. Experiments were repeated for three times and the data showed the representative results. (C, D) Cell viability was detected by CCK-8 assay after A549 (C) and NCI-H226 (D) cell lines treated with combination of Sunitinib or R8 and autophagy inhibitor 3-MA (5 $\mathrm{mM}$ ) for $48 \mathrm{~h}$. 
glioblastoma, melanoma [12-15] by inhibiting the activation of VEGFR-1, 2 and 3, PDGFR- $\alpha, \beta$, Kit, Flt3 and RET. It has been approved by FDA to treat advanced renal cell cancer and imatinib mesylate-intolerant or resistant gastrointestinal stromal tumor since 2006 [16, 17].

Our results showed that R8 is more potent than Sunitinib in suppression of all types of cancer cells tested in this study, with much lower $\mathrm{IC}_{50}$ values when compared to Sunitinib. Moreover, when specifically focused on lung cancer, one type of cancer with high morbidity and mortality, R8 exhibits stronger inhibition than Sunitinib on colony formation, cell cycle arrest and migration of lung cancer cells A549 and NCI-H226, although less effective than Sunitinib in inhibiting tube formation of endothelial cells. PI3K-Akt-mTOR has been identified as a key signal pathway in regulating various aspects such as cancer cell proliferation, migration, apoptosis and autophagy [18, 19], and in this study we did observed that R8 induced significant down-regulation of p-AKT and total-Akt than Sunitinib did. Because of its key role in promoting cell cycle from G1 to S phase, down-regulated p-AKT resulted in cell cycle arrest at $\mathrm{G} 0 / \mathrm{G} 1$ phase and proliferation inhibition.

Suppression of the Akt/mTOR pathway may also trigger autophagy and apoptosis [20-22]. Autophagy represents an important cell physiologic process in response to starvation, hypoxia, ER stress and oxidative stress. Autophagosomes engulf cellular organelles, such as mitochondria, ribosome, then fuse with lysosome to recycle energy used for cell survival during stress [23]. Generally, autophagy is considered as a way to protect cells from apoptosis [24-26]. Nonetheless, if stress over a safe threshold, autophagy can lead to cell death, called autophagic cell death or type II programmed cell death [27-29]. Indeed, several studies have confirmed the complex relationship between apoptosis and autophagy during the progress of cell treated with chemotherapeutic agents [30-33]. In this study we checked apoptosis and autophagy status of cancer cells treated with Sunitinib or R8 after $48 \mathrm{~h}$. Because of down-regulation of PI3KAkt-mTOR pathway, R8 induced significantly autophagy than Sunitinib in a dose-dependent manner marked by accumulation of LC3-II. Bcl-2 family proteins were activated to trigger apoptosis, resulted in up-regulation of Bax, cleavage of caspase-3 and PARP [34]. In the current study, we observed that suppression of R8-induced autophagy resulted in attenuated cleavage of caspase- 3 and PARP, while the cleavage of caspase- 3 and PARP were elevated on inhibition of Sunitinib-induced autophagy. The different sensitivities to 3-MA and CQ observed in this study were due to different mechanisms the two inhibitors involved in autophagy. Although both 3-MA and CQ are autophagy inhibitors, they block different steps of autophagy. 3-MA suppresses an early stage of autophagy by inhibiting the class III PI3K to block the production of PI3P, which is essential for early autophagosome formation [35]. CQ is a lysosomotropic agent which blocks the late stage of autophagy by elevating the lysosomal $\mathrm{pH}$, inhibiting autophagosome fusion with lysosome and autophagosome degradation [36]. Taken together, these results suggested that Sunitinib-induced a pro-survival autophagy to evade cytotoxicity in lung cancer cells, similar to that in ovarian cancer [37], whereas R8-induced autophagy may have synergetic effect on cell death with apoptosis.

Our results also showed some differences between A549 and NCI-H226. NCI-H226, with more abundant receptor tyrosine kinases, showed more resistant to TKIs with higher $\mathrm{IC}_{50}$ than $\mathrm{A} 549$, while $\mathrm{A} 549$, with less hyperactivated tyrosine kinase receptors, was more sensitive to R8. Interestingly, we found that the inhibition of EGFR phosphorylation in A549 was not only independent of the dose of R8, but even showed up-regulation of p-EGFR when treated with higher dose of $\mathrm{R} 8$. Whether this is just a feedback of self-protective response to the severe stress or by some other specific mechanisms need to be investigated in the future. Furthermore, the differences between the two cell lines on critical steps during autophagy may also be due to the different responses to RTK inhibition. For A549, EGFR is a prominent tyrosine kinase. Activated EGFR phosphorylates Beclin-1 at three different tyrosine residues, Y229, Y233 and Y352, provokes the association of Beclin-1 with Rubicon/Bcl-2, which results in reduced autophagy. Exposure of A549 to tyrosine kinase inhibitors restores the Beclin-1-VPS34 interaction and triggers an increase in autophagy, but it is independent of mTOR, modulation of the Beclin-1-Rubicon/Bcl-2 complex is dominant in the EGFR-mediated autophagy. In NCI-H226 cell line, PDGFR can not induce Beclin-1 phosphorylation, but can strongly activate autophagy by the PI3K-mTOR pathway [38-40]. Thus, the mechanism of autophagy regulation, by EGFR-Beclin-1 or PI3K-mTOR, may be an important factor to decide critical steps in cell fate.

Notably, we demonstrated in this work that R8 can significantly inhibit the phosphorylation of PDGFR $\beta$ and VEGFR2. Previous studies have shown the association between dysregulation of these two RTKs and chemoresistance: increased PDGFR $\beta$ and VEGFR2 protein levels were associated with resistance to platinumbased chemotherapy and adverse outcome of ovarian cancer patients [41]; increased VEGFR2 gene copy was associated with chemo-resistance and shorter survival in non-small cell lung cancer patients who received platinum adjuvant chemotherapy after surgical resection [42]. Moreover, pretreatment with VEGFR and PDGFR $\beta$ inhibitors can inhibit tumor growth in a mouse colorectal carcinomatosis model [43]. Therefore, malignancies with dysregulated PDGFR $\beta$ and VEGFR2 may benefit from R8 treatment due to the potent inhibition on these RTKs.

In conclusion, current work showed that the novel compound $\mathrm{R} 8$ is a potent multikinase inhibitor which 
can effectively inhibit the phosphorylation of PDGFR $\beta$, VEGFR2, EGFR and C-Kit. R8 shows more in vitro suppression on lung cancer cells than Sunitinib does by the synergetic effect of autophagic cell death and apoptosis. This provides the potential that $\mathrm{R} 8$ alone may serve as a good chemotherapeutic drug without co-administration of an autophagy inhibition drug to enhance the therapeutic efficacy which is suggested for many chemotherapeutic drugs. Further in vivo assays such as non-small cell lung cancer patient-derived xenograft models which express high level of PDGFR $\beta$, VEGFR2, EGFR, C-Kit will be carried out to demonstrate that whether $\mathrm{R} 8$ can be developed into a new member of MKI drug family.

\section{MATERIALS AND METHODS}

\section{Chemicals and reagents}

Sunitinib (PubChem CID: 6456015, purity: 99.64\%) was from Nanjing First Pharmaceutical Co., Ltd (Nanjing, China), R8 was synthesized by WuXi AppTec (Shanghai, China). 3-Methyladenine (3-MA, PubChem CID: 1673) was purchased from Selleck Chemicals (Houston, Texas, USA), Chloroquine Phosphate (CQ, PubChem CID: 64927) was purchased from Sigma (St. Louis, MO, USA).

Cell counting kit-8 was obtained from Dojindo (Tokyo, Japan). PI/RNase Staining Buffer was obtained from BD Bioscience (San Diego, CA, USA). Matrigel ${ }^{\circledR}$ Basement Membrane Matrix was obtained from Corning (Corning, NY, USA). Dc Protein assay kit and TGX Stain-free FastCast acrylamide Kit was purchased from Bio-Rad (Hercules, CA, USA). Complete ${ }^{\mathrm{TM}}$ Protease Inhibitor Cocktail Tablet and Phosphatase Inhibitor Cocktail 2 were purchased from Santa Cruze (Santa Cruz, CA, USA) and Sigma (St. Louis, MO, USA), respectively. BSA was purchased from Amresco (Solon, $\mathrm{OH}$, USA). Immobilon ${ }^{\mathrm{TM}}$ western chemiluminescent HRP substrate was purchased from Millipore (Billerica, MA, USA). Antibodies for Phospho-PDGF Receptor $\beta$ (Tyr751), PDGF Receptor $\beta$, Phospho-VEGF Receptor 2 (Tyr1175), VEGF Receptor 2, Phospho-C-Kit (Tyr719), C-Kit, Phospho-EGF Receptor (Tyr1068), PhosphoAkt (Ser473), Akt, Phospho-p44/42 MAPK (Erk1/2) (Thr202/Tyr204), p44/42 MAP Kinase, Bax, PARP, LC3 and Beclin-1 were all purchased from Cell signaling technology (Beverly, MA, USA). Antibody against EGFR was purchased from Abcam (Cambridge, MA, USA). HRP-conjugated Monoclonal Mouse Anti-GAPDH was purchased from KangChen Biotech (Shanghai, China). Secondary antibodies were purchased from Santa Cruz Biotechnology (Santa Cruz, CA, USA).

\section{Cell culture}

Human gastric carcinoma cells SGC7901, non-small cell lung cancer cell line A549 and NCI-H226, laryngeal carcinoma cell line HEp-2, renal adenocarcinoma cell line 786-0, colorectal cancer cell line SW620 were obtained from American Type Culture Collection (Manassas, VA, USA). Cells were maintained in RPMI-1640 medium, supplemented with $10 \%$ fetal bovine serum (FBS) at $37^{\circ} \mathrm{C}$ in a humidified incubator with $5 \% \mathrm{CO}_{2}$, Primary human umbilical vein endothelial cell line (HUVEC) was a kind gift from Professor Jianan Wang (Zhejiang University School of Medicine, China).

\section{Cell viability assay}

$\mathrm{R} 8$ and Sunitinib were dissolved in $\mathrm{ddH}_{2} \mathrm{O}$ or RPMI-1640 medium respectively, to give a storage concentration of $20 \mathrm{mM}$ and stored at $-20^{\circ} \mathrm{C}$ for no more than one week. For A549, cells were seeded at a density of $8 \times 10^{3}$ per well in 96-well plate and cultured for 24 h. After 24 h, 10\% FBS-RPMI1640 was removed, cells were starved overnight. Then, cells were exposed to different concentration of $\mathrm{R} 8$ or Sunitinib (range from $2.5 \mu \mathrm{M}-80 \mu \mathrm{M})$ for $1 \mathrm{~h}$ before stimulation. Subsequently, cells were stimulated with same volume of $1 \%$ FBSRPMI1640 and cultured in the presence of R8 or Sunitinib. After 48 h, cell viability was assessed by cell counting kit-8 assay as described previously [44]. The $\mathrm{IC}_{50}$ of $\mathrm{R} 8$ and Sunitinib were calculated based on the cell viability curve.

\section{Cell cycle assay}

A549 $\left(4 \times 10^{5} /\right.$ well $)$ and NCI-H226 $\left(5 \times 10^{5} /\right.$ well $)$ cells were seeded in 6-well plate and cultured for $24 \mathrm{~h}$. All procedures were the same as cell viability assay except for that we harvested the cells at $24 \mathrm{~h}$ after stimulation. Then cells were fixed with $75 \%$ ethanol overnight followed by RNase treatment and propidium iodide (PI) staining as the instruction of PI/RNase Staining Buffer indicated. Cells were subjected to flow cytometer (FACSCalibur, BD, San Jose, CA, USA) and analyzed using CELL Quest 3.0 software (BD, NJ, USA).

\section{Colony formation assay}

A549 (500/well) and NCI-H226 (800/well) cells were seeded in 6-well plate and cultured for $24 \mathrm{~h}$. After serum-starved overnight, cells were exposed to different concentrations of $\mathrm{R} 8$ and Sunitinib for $1 \mathrm{~h}$ then stimulated with same volume of 10\% FBS-RPMI1640. After that, cells were cultured for 14 days to allow colony formation. Colonies were fixed with 4\% PFA for 15 min then stained with $1 \%$ crystal violet for 15 min. Colony consisting of 100 or more cells was scored [45]. The results were photographed by ChemiDoc XRS+ image system of Bio-Rad (Hercules, CA, USA) and Lecia DMIL LED inverted microscope (Wetzlar, Germany). 


\section{Migration assay}

A549 $\left(6 \times 10^{5} /\right.$ well $)$ and NCI-H226 $\left(5 \times 10^{5} /\right.$ well $)$ were seeded in 6-well plate and cultured for $24 \mathrm{~h}$. After that medium was replaced by RPMI-1640 medium without FBS, cells were starved for $24 \mathrm{~h}$. Then on the third day, $10 \mu l$ pipette tip was used to scribe gaps in each well. Scratched cells were washed with PBS for three times. Simultaneously, the gaps were photographed, set as $0 \mathrm{~h}$. Finally, different concentrations of R8 and Sunitinib were added into the medium. Photographs were captured at 12, $24,36 \mathrm{~h}$ by inverted microscope for the same position [46]. The migrated distances were analyzed by Image-pro plus software.

\section{Tube formation assay}

Matrigel basement membrane matrix was placed in $4^{\circ} \mathrm{C}$ overnight before use. On the following day, a 96-well plate was coated with $50 \mu 1$ Matrigel on ice and incubated at $37^{\circ} \mathrm{C}$ for $30 \mathrm{~min}$. Then HUVECs $\left(10^{4} /\right.$ well) were added in serum-free medium in the absence or presence of various concentrations of R8 or Sunitinib. Each conditional group contained 5 wells. Pictures of representative $\times 10$ fields were taken from 4 to $6 \mathrm{~h}$. The length of endothelial tubes was measured by Image-Pro Plus to assess the tube formation ability.

\section{Western blot analysis}

Cells were seeded in $100 \mathrm{~mm}$ culture dishes at a density of $1.5 \times 10^{6}$ per dish. To evaluate the function of R8 on inhibiting growth factor stimulated receptor phosphorylation, cells were exposed to R8 for $1 \mathrm{~h}$ then harvested immediately on ice after simulated with $10 \%$ FBS-RPMI 1640 for $10 \mathrm{~min}$. To evaluate autophagy and apoptosis, cells were exposed to R8 and Sunitinib for 1 $\mathrm{h}$ then stimulated with 1\% FBS-RPMI1640. Cells were harvested after $48 \mathrm{~h}$ treatment. For autophagy inhibitor, cells were treated with $3-\mathrm{MA}(10 \mathrm{mM})$ or CQ $(20 \mu \mathrm{M})$ for $2 \mathrm{~h}$ before exposed to R8 and Sunitinib.

The cell pellets were lysed in RIPA lysis buffer containing 100× Phosphatase Inhibitor Cocktail 2 and $50 \times$ Complete $^{\mathrm{TM}}$ Protease Inhibitor Cocktail for $1 \mathrm{~h}$ on ice. Cell lysates were centrifuged at $14,000 \times \mathrm{g}$ for 15 min at $4^{\circ} \mathrm{C}$ then supernatant extracts were collected. The protein concentration was determined by Dc Protein assay kit of Bio-Rad. Equal amount of proteins $(30 \mu \mathrm{g} /$ well) were separated by electrophoresis on $10-12 \%$ SDSPAGE and transferred to PVDF membranes from BioRad (Hercules, CA, USA). Membranes were blocked with $10 \%$ BSA-TBST for $1 \mathrm{~h}$ at room temperature and then incubated with primary antibodies at $4^{\circ} \mathrm{C}$ overnight. Next, membranes were incubated with an HRP-conjugated secondary antibody for $1 \mathrm{~h}$ at room temperature. The immune-reactive bands were visualized by the ECL kit from Millipore (Billerica, MA, USA).

\section{Statistical analysis}

Each experiment was performed at least for three times, and the data were shown as means \pm standard deviation. The statistic difference was analyzed by twotailed Student's $t$-test. $P$ values $<0.05$ were considered to be statistically significant.

\section{Abbreviations}

PDGFR: platelet-derived growth factor receptor; VEGFR: vascular endothelial growth factor receptor; EGFR: epidermal growth factor receptor; RTK: receptor tyrosine kinase; TKI: tyrosine kinase inhibitor; MKI: multi-target tyrosine kinase inhibitor; GIST: gastrointestinal stromal tumor; $\mathrm{IC}_{50}$ : median inhibitory concentration; PCD: programmed cell death.

\section{Author contributions}

YX, JC participated in research design; YX, CL conducted experiments; $\mathrm{YH}, \mathrm{ZJ}$ contributed new reagents and analytic tools; YX performed data analysis; YX, JC contributed to the writing of the manuscript.

\section{ACKNOWLEDGMENTS}

We are grateful for the technical support provided by Ms. Lingyan Wu (Clinical Research Center, The Second Affiliated Hospital, Zhejiang University School of Medicine) on flow cytometric analysis.

\section{CONFLICTS OF INTEREST}

The authors declare that there are no conflicts of interest.

\section{FUNDING}

This work was supported by the National Natural Science Foundation of China [30271450, 30471955 and 30672365]; and the Natural Science Foundation of Department of Science and Technology of Zhejiang Province [2013F10052].

\section{REFERENCES}

1. Torre LA, Bray F, Siegel RL, Ferlay J, Lortet-Tieulent J, Jemal A. Global cancer statistics, 2012. CA Cancer J Clin. 2015; 65:87-108.

2. Gentile C, Martorana A, Lauria A, Bonsignore R. Kinase inhibitors in multitargeted cancer therapy. Curr Med Chem. 2017; 24:1671-1686.

3. Husain H, Scur M, Murtuza A, Bui N, Woodward B, Kurzrock R. Strategies to overcome bypass mechanisms mediating clinical resistance to EGFR tyrosine kinase 
inhibition in lung cancer. Mol Cancer Ther. 2017; 16:265-272.

4. Santarpia M, Gil N, Rosell R. Strategies to overcome resistance to tyrosine kinase inhibitors in non-small-cell lung cancer. Expert Rev Clin Pharmacol. 2015; 8:461-477.

5. Chang J, Wang S, Zhang Z, Liu X, Wu Z, Geng R, Ge X, Dai C, Liu R, Zhang Q, Li W, Li J. Multiple receptor tyrosine kinase activation attenuates therapeutic efficacy of the fibroblast growth factor receptor 2 inhibitor AZD4547 in FGFR2 amplified gastric cancer. Oncotarget. 2015; 6:20092022. https://doi.org/10.18632/oncotarget.2987.

6. Tannir NM, Schwab G, Grunwald V. Cabozantinib: an active novel multikinase inhibitor in renal cell carcinoma. Curr Oncol Rep. 2017; 19:14.

7. Hewett Y, Ghimire S, Farooqi B, Shah BK. Lenvatinib - a multikinase inhibitor for radioiodine-refractory differentiated thyroid cancer. J Oncol Pharm Pract. 2016.

8. Majithia N, Grothey A. Regorafenib in the treatment of colorectal cancer. Expert Opin Pharmacother. 2016; 17:137-145.

9. Tanday S. Sunitinib benefits patients with small-cell lung cancer. Lancet Oncol. 2015; 16:e164.

10. Baggstrom MQ, Socinski MA, Wang XF, Gu L, Stinchcombe TE, Edelman MJ, Baker S Jr, Feliciano J, Novotny P, Hahn O, Crawford JA, Vokes EE. Maintenance sunitinib following initial platinum-based combination chemotherapy in advanced-stage IIIB/IV non-small cell lung cancer: a randomized, double-blind, placebo-controlled phase III study-CALGB 30607 (Alliance). J Thorac Oncol. 2017; 12:843-849.

11. Hao F, Wang S, Zhu X, Xue J, Li J, Wang L, Li J, Lu W, Zhou T. Pharmacokinetic-pharmacodynamic modeling of the anti-tumor effect of sunitinib combined with dopamine in the human non-small cell lung cancer xenograft. Pharm Res. 2017; 34:408-418.

12. Motzer RJ, Rini BI, Bukowski RM, Curti BD, George DJ, Hudes GR, Redman BG, Margolin KA, Merchan JR, Wilding G, Ginsberg MS, Bacik J, Kim ST, et al. Sunitinib in patients with metastatic renal cell carcinoma. JAMA. 2006; 295:2516-2524.

13. Abrams TJ, Lee LB, Murray LJ, Pryer NK, Cherrington JM. SU11248 inhibits KIT and platelet-derived growth factor receptor beta in preclinical models of human small cell lung cancer. Mol Cancer Ther. 2003; 2:471-478.

14. Potapova O, Laird AD, Nannini MA, Barone A, Li G, Moss KG, Cherrington JM, Mendel DB. Contribution of individual targets to the antitumor efficacy of the multitargeted receptor tyrosine kinase inhibitor SU11248. Mol Cancer Ther. 2006; 5:1280-1289.

15. Andrae N, Kirches E, Hartig R, Haase D, Keilhoff G, Kalinski T, Mawrin C. Sunitinib targets PDGF-receptor and Flt3 and reduces survival and migration of human meningioma cells. Eur J Cancer. 2012; 48:1831-1841.
16. Socinski MA. Multitargeted receptor tyrosine kinase inhibition: an antiangiogenic strategy in non-small cell lung cancer. Cancer Treat Rev. 2011; 37:611-617.

17. Wakelee HA, Schiller JH. Targeting angiogenesis with vascular endothelial growth factor receptor small-molecule inhibitors: novel agents with potential in lung cancer. Clin Lung Cancer. 2005; 7:S31-38.

18. Liu J, Liu L, Xue Y, Meng F, Li S, Wang P, Liu Y. Antineoplastic activity of low-dose endothelial-monocyte activating polypeptide-II results from defective autophagy and $\mathrm{G} 2 / \mathrm{M}$ arrest mediated by PI3K/Akt/FoxO1 axis in human glioblastoma stem cells. Biochem Pharmacol. 2014; 89:477-489.

19. Saini KS, Loi S, de Azambuja E, Metzger-Filho O, Saini ML, Ignatiadis M, Dancey JE, Piccart-Gebhart MJ. Targeting the PI3K/AKT/mTOR and Raf/MEK/ERK pathways in the treatment of breast cancer. Cancer Treat Rev. 2013; 39:935-946.

20. Polivka J Jr, Janku F. Molecular targets for cancer therapy in the PI3K/AKT/mTOR pathway. Pharmacol Ther. 2014; 142:164-175.

21. Hanahan D, Weinberg RA. Hallmarks of cancer: the next generation. Cell. 2011; 144:646-674.

22. Gurpinar E, Grizzle WE, Shacka JJ, Mader BJ, Li N, Piazza NA, Russo S, Keeton AB, Piazza GA. A novel sulindac derivative inhibits lung adenocarcinoma cell growth through suppression of Akt/mTOR signaling and induction of autophagy. Mol Cancer Ther. 2013; 12:663-674.

23. Kenific CM, Debnath J. Cellular and metabolic functions for autophagy in cancer cells. Trends Cell Biol. 2015; 25:37-45.

24. Viola G, Bortolozzi R, Hamel E, Moro S, Brun P, Castagliuolo I, Ferlin MG, Basso G. MG-2477, a new tubulin inhibitor, induces autophagy through inhibition of the Akt/mTOR pathway and delayed apoptosis in A549 cells. Biochem Pharmacol. 2012; 83:16-26.

25. Martin S, Dudek-Peric AM, Maes H, Garg AD, Gabrysiak M, Demirsoy S, Swinnen JV, Agostinis P. Concurrent MEK and autophagy inhibition is required to restore cell death associated danger-signalling in Vemurafenib-resistant melanoma cells. Biochem Pharmacol. 2015; 93:290-304.

26. Xie N, Zhong L, Liu L, Fang Y, Qi X, Cao J, Yang B, He Q, Ying M. Autophagy contributes to dasatinib-induced myeloid differentiation of human acute myeloid leukemia cells. Biochem Pharmacol. 2014; 89:74-85.

27. Tsujimoto Y, Shimizu S. Another way to die: autophagic programmed cell death. Cell Death Differ. 2005; 12:1528-1534.

28. Bhutia SK, Mukhopadhyay S, Sinha N, Das DN, Panda PK, Patra SK, Maiti TK, Mandal M, Dent P, Wang XY, Das SK, Sarkar D, Fisher PB. Autophagy: cancer's friend or foe? Adv Cancer Res. 2013; 118:61-95.

29. Liu R, Li J, Zhang T, Zou L, Chen Y, Wang K, Lei Y, Yuan K, Li Y, Lan J, Cheng L, Xie N, Xiang R, et al. Itraconazole 
suppresses the growth of glioblastoma through induction of autophagy: involvement of abnormal cholesterol trafficking. Autophagy. 2014; 10:1241-1255.

30. Radogna F, Dicato M, Diederich M. Cancer-type-specific crosstalk between autophagy, necroptosis and apoptosis as a pharmacological target. Biochem Pharmacol. 2015; 94:1-11.

31. Yuan N, Song L, Zhang S, Lin W, Cao Y, Xu F, Fang Y, Wang Z, Zhang H, Li X, Wang Z, Cai J, Wang J, et al. Bafilomycin A1 targets both autophagy and apoptosis pathways in pediatric B-cell acute lymphoblastic leukemia. Haematologica. 2015; 100:345-356.

32. Santoni M, Amantini C, Morelli MB, Liberati S, Farfariello V, Nabissi M, Bonfili L, Eleuteri AM, Mozzicafreddo M, Burattini L, Berardi R, Cascinu S, Santoni G. Pazopanib and sunitinib trigger autophagic and non-autophagic death of bladder tumour cells. Br J Cancer. 2013; 109:1040-1050.

33. Ojha R, Ishaq M, Singh SK. Caspase-mediated crosstalk between autophagy and apoptosis: mutual adjustment or matter of dominance. J Cancer Res Ther. 2015; 11:514-524.

34. Aliwaini S, Swarts AJ, Blanckenberg A, Mapolie S, Prince S. A novel binuclear palladacycle complex inhibits melanoma growth in vitro and in vivo through apoptosis and autophagy. Biochem Pharmacol. 2013; 86:1650-1663.

35. Wu YT, Tan HL, Shui G, Bauvy C, Huang Q, Wenk MR, Ong CN, Codogno P, Shen HM. Dual role of 3-methyladenine in modulation of autophagy via different temporal patterns of inhibition on class I and III phosphoinositide 3-kinase. J Biol Chem. 2010; 285:10850-10861.

36. Klionsky DJ, Abdelmohsen K, Abe A, Abedin MJ, Abeliovich H, Acevedo Arozena A, Adachi H, Adams CM, Adams PD, Adeli K, Adhihetty PJ, Adler SG, Agam G, et al. Guidelines for the use and interpretation of assays for monitoring autophagy (3rd edition). Autophagy. 2016; 12:1-222.

37. DeVorkin L, Hattersley M, Kim P, Ries J, Spowart J, Anglesio MS, Levi SM, Huntsman DG, Amaravadi RK, Winkler JD, Tinker AV, Lum JJ. Autophagy inhibition enhances sunitinib efficacy in clear cell ovarian carcinoma. Mol Cancer Res. 2017; 15:250-258.

38. Wei Y, Zou Z, Becker N, Anderson M, Sumpter R, Xiao G, Kinch L, Koduru P, Christudass CS, Veltri RW, Grishin
NV, Peyton M, Minna J, et al. EGFR-mediated Beclin 1 phosphorylation in autophagy suppression, tumor progression, and tumor chemoresistance. Cell. 2013; 154:1269-1284.

39. Tan X, Thapa N, Sun Y, Anderson RA. A kinaseindependent role for EGF receptor in autophagy initiation. Cell. 2015; 160:145-160.

40. Fantin VR, Abraham RT. Self-eating limits EGFRdependent tumor growth. Cell. 2013; 154:1184-1186.

41. Avril S, Dincer Y, Malinowsky K, Wolff C, Gundisch S, Hapfelmeier A, Boxberg M, Bronger H, Becker KF, Schmalfeldt B. Increased PDGFR-beta and VEGFR-2 protein levels are associated with resistance to platinumbased chemotherapy and adverse outcome of ovarian cancer patients. Oncotarget. 2017; 154:1269-1284. https://doi. org/10.18632/oncotarget.18415..

42. Yang F, Tang X, Riquelme E, Behrens C, Nilsson MB, Giri U, Varella-Garcia M, Byers LA, Lin HY, Wang J, Raso MG, Girard L, Coombes K, et al. Increased VEGFR-2 gene copy is associated with chemoresistance and shorter survival in patients with non-small-cell lung carcinoma who receive adjuvant chemotherapy. Cancer Res. 2011; 71:5512-5521.

43. Gremonprez F, Descamps B, Izmer A, Vanhove C, Vanhaecke F, De Wever O, Ceelen W. Pretreatment with VEGF(R)-inhibitors reduces interstitial fluid pressure, increases intraperitoneal chemotherapy drug penetration, and impedes tumor growth in a mouse colorectal carcinomatosis model. Oncotarget. 2015; 6:29889-29900. https://doi.org/10.18632/oncotarget.5092.

44. Xie Y, Li Q, Yang Q, Yang M, Zhang Z, Zhu L, Yan H, Feng R, Zhang S, Huang C, Liu Z, Wen T. Overexpression of DCF1 inhibits glioma through destruction of mitochondria and activation of apoptosis pathway. Sci Rep. 2014; 4:3702.

45. Yoon C, Park DJ, Schmidt B, Thomas NJ, Lee HJ, Kim TS, Janjigian YY, Cohen DJ, Yoon SS. CD44 expression denotes a subpopulation of gastric cancer cells in which Hedgehog signaling promotes chemotherapy resistance. Clin Cancer Res. 2014; 20:3974-3988.

46. Liang CC, Park AY, Guan JL. In vitro scratch assay: a convenient and inexpensive method for analysis of cell migration in vitro. Nat Protoc. 2007; 2:329-333. 